

\section{COMPRESSION ALGORITHM}

There are two types of compression algorithms are Lossless and Lossy. The packed picture is absolutely copy of the first info image in the misfortune less compressed, so there is no measure of loss present in the image. Be that as it may, in Lossy compression the compressed image is not same as the information image, some measure of loss is available in the image.

\subsection{Lossless image compression}

In lossless compression plan recreated image is same to the input image. Lossless image compression methods first change over the image into the image pixels. At that point handling is done on every single pixel. The First step incorporates expectation of next picture pixel esteem from the area pixels. In the second stage the distinction between the predicted valie and the actual intensity of following pixel is coded utilizing diverse encoding techniques.

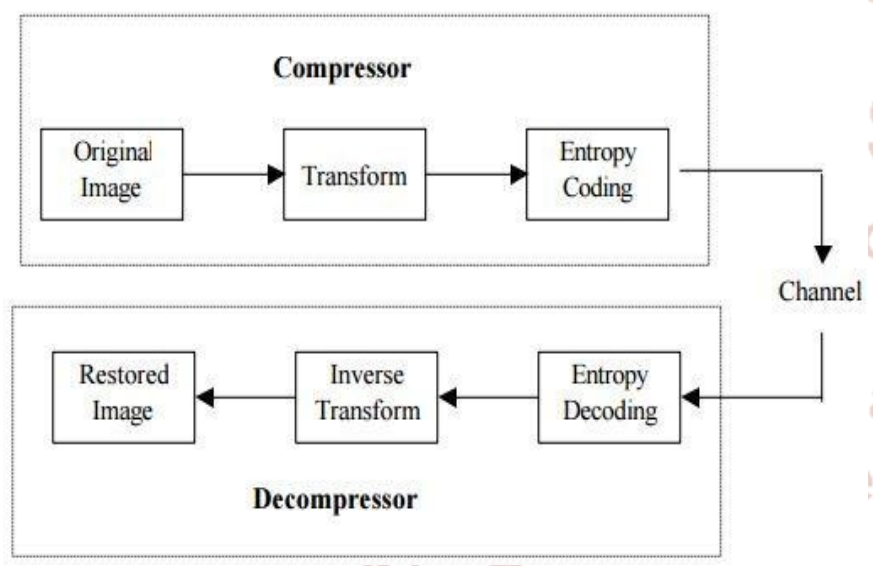

Fig.1 Block diagram of Lossless compression method

\subsection{Lossy Compression Techniques}

Lossy compression technique provides higher pressure proportion contrast with lossless compression. In this strategy, the compressed image is not same as the first picture; there is some measure of data loss in the image. Lossy compression method is appeared in fig.

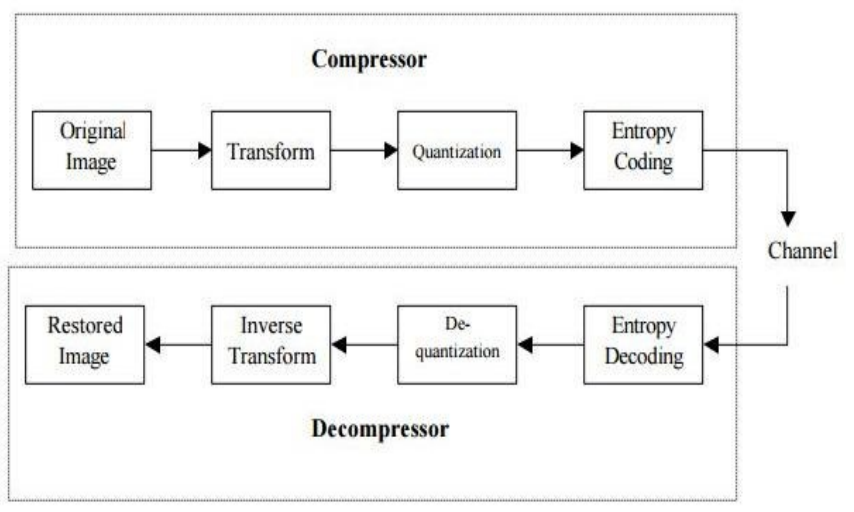

Fig.2 Block diagram of Lossy compression method

\section{PURPOSE OF IMAGE COMPRESSION}

Size of picture can be minimized utilizing Image compression technique strategy as a part of bytes of an illustrations without debasing the nature of the image to an unsatisfactory level. The diminishment in record size stores more images in a given measure of plate or memory space. The decrease in document estimate additionally lessens the time required for images to be sent over the web or downloaded from Web pages. There are a few distinctive courses in which image records can be compacted. The two most regular compacted realistic image arrangements are the JPEG group and the GIF design. The JPEG method is utilized for photos, while the GIF method is commonly used for line art and other images in which geometric shapes are relatively simple. Different systems for image compression incorporate the utilization of fractals and wavelets advancements. Both these two advances offer higher compression ratio as compared to the JPEG or GIF methods. Another new strategy is the PNG format. A content document or program can be compacted without the presentation of mistakes, however just up to a specific degree or up to great level.

\section{CIMETHODS USED IN IMAGE COMPRESSION}

\subsection{Embedded Zerotree Wavelet (EZW)}

Embedded Zerotree Wavelet is a lossy image compression algorithm. At low bit rates, i.e. high compression ratios, the vast majority of the coefficients delivered by a sub band transform will be zero, or near zero [6]. This happens on the grounds that "true " images have a tendency to contain for the most part low frequency data. However where high frequency data occurs great coding plan is utilized. In zerotree based image compression plan, for example, EZW and SPIHT, accentuation on the utilization of measurable properties of the trees keeping in mind the end goal to code the areas of the huge coefficients proficiently [1]. Since the most of the coefficients will be zero or near zero, the spatial areas of the noteworthy coefficients make up a huge bit of the aggregate size of a commonplace compacted image. A coefficient is viewed as a critical if its extent is over a specific limit. By beginning with a limit which is near the maximum coefficient magnitude and iteratively decreasing the threshold, it is conceivable to make a compressed representation of a image which continuously includes better detail. Because of the structure of the trees, it is likely that if a coefficient in 
a specific frequency band is inconsequential, then every one of its relatives will likewise be immaterial.

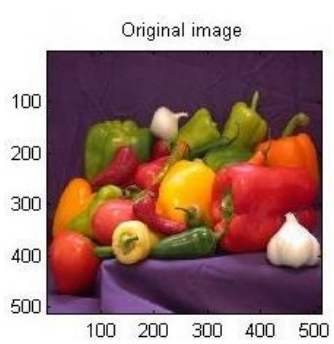

SIZE (150 KB)

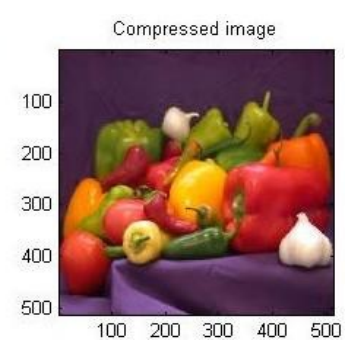

SIZE (52 KB)
Fig.3 Image compression using EZW

\subsection{Wavelet Difference Reduction (WDR)}

The WDR consolidates run-length coding of the centrality map with an effective representation of the run length images to deliver an embedded image coder. In both SPIHT and WDR systems, the zero tree data structure is precluded, however the emmbedded principal of lossless bit plane coding and set apportioning are protected. In the WDR algorithm, rather than utilizing the zero trees, every coefficient in a decomposed wavelet pyramid is allocated a straight position list. The output of the WDR encoding can be arithmetically. The method that they describe is based on the elementary arithmetic coding algorithm. The WDR algorithm is a very simple procedure. A wavelet transform is first applied to the picture, and then the bit-plane based WDR encoding calculation for the wavelet coefficients is completed.

\subsection{Adaptively Scanned Wavelet Difference} Reduction (ASWDR)

A standout amongst the latest image compression algorithm is the Adaptively Scanned Wavelet contrast Reduction (ASWDR) calculation of Walker. The descriptor adaptively checked alludes to the way that this calculation alters the examining request utilized by WDR as a part of request to accomplish better execution

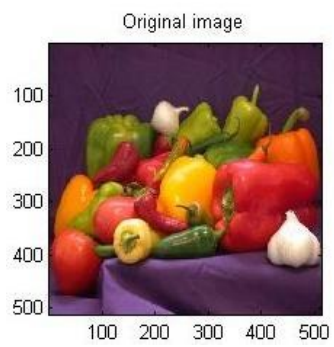

Size (150 Kb)

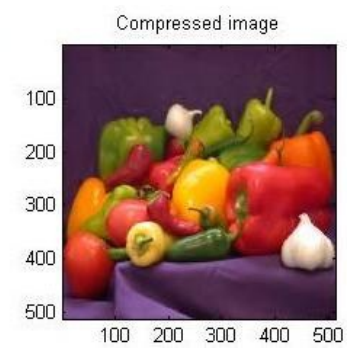

Size (46 Kb)
Fig.4 Image compression using ASWDR
5.4 Set Partitioning In Hierarchical Trees 3D for True color Images (SPIHT_3D)

The proposed algorithm shows a use of 3D-SPIHT algorithm to color volumetric dicom medicinal pictures utilizing 3D wavelet decay and a 3D spatial dependence tree [3]. The wavelet decomposition is expert with biorthogonal 9/7 filters [2]. 3D-SPIHT is the cutting edge benchmark for three dimensional image compressions. The three-dimensional coding is based on the observation that the sequences of images are contiguous in the temporal axis and there is no motion between cuts . i.e., the 3D discrete wavelet transform can fully exploit the inter-slices correlations [11]. The set dividing strategies include a progressive coding of the wavelet coefficients. The 3D SPIHT is executed and the Rate-mutilation (Peak Signal-toNoise Ratio (PSNR) versus bit rate) exhibitions are displayed for volumetric therapeutic datasets by utilizing bi orthogonal $9 / 7$. The results are compared to previous result of JPEG 2000 standards. Results shows that 3D-SPIHT technique abuses the color space connections and keeping up the full embeddedness required by color image sequence compression and gives better execution as far as the PSNR and compression ratio than the JPEG 2000. The results suggest the effective practical for PACS applications.
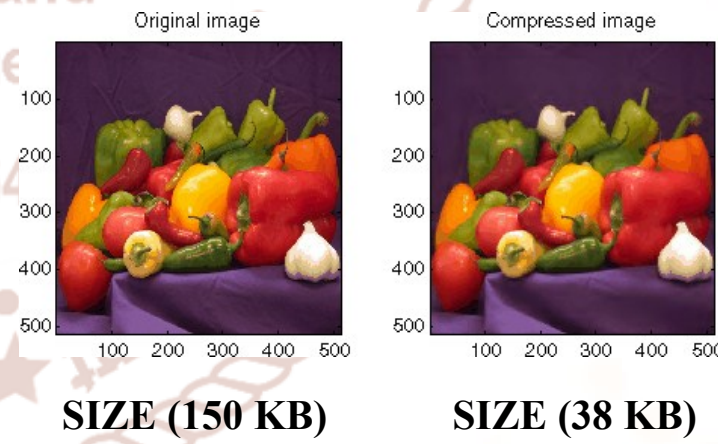

Fig.5 Image compression using SPIHT (True colour)

\subsection{Set Partitioning In Hierarchical Trees (SPIHT)}

The images got with wavelet-based strategies yield great visual quality. Indeed, even basic coding strategies delivered great results when consolidated with wavelets. SPIHT has a place with the up and coming era of wavelet encoders, employing more sophisticated coding. SPIHT exploits the properties of the wavelet-transfered images to increase its efficiency [5]. SPIHT wins in the trial of finding the base rate required to get a generation indistinct from the first. The SPIHT favorable position is considerably more professed in encoding color images, in light of the fact that the bits are 
apportioned consequently for nearby optimality among the color parts, not at all like different algorithm that encode the color components independently in light of worldwide measurements of the Individual components.

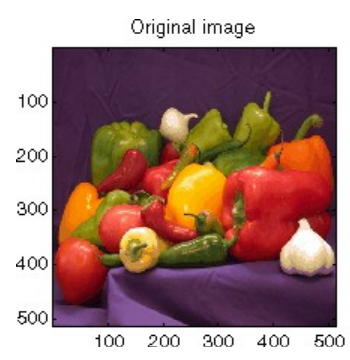

SIZE (150 KB)

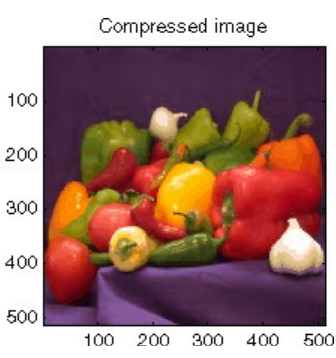

SIZE (26 KB)
Fig.6 Image compression using SPIHT (Gray colour)

\subsection{Spatial-orientation Tree Wavelet (STW)}

Spatial introduction trees, are gatherings of wavelet transform coefficients composed into trees with lowest frequency sub band is the root and higher frequency sub bands are with the offspring in the lowest frequency or coarsest scale sub band is with the offspring . 3D-SPIHT: The developed version of 2D SPIHT is the 3-D SPIHT plan having the same three characteristics. 1) By arranging partially with the magnitude of the 3-D wavelet transformed video using a 3-D set partitioning algorithm; 2) transmission of refinement bits in an ordered bit plane; and 3) utilization of self-similarity across spatial-temporal orientation.

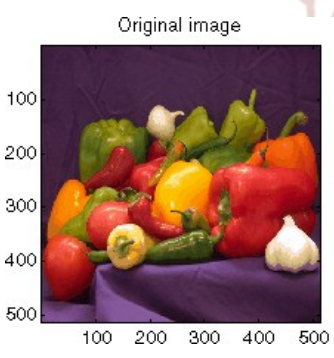

SIZE (150 KB)

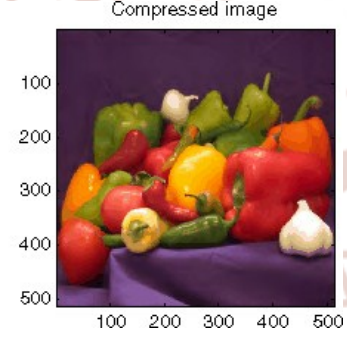

SIZE (23.4KB)
Fig.7Image Compression Using STW
Table 2. Comparison chart of these algorithms are given below:

\begin{tabular}{|c|c|c|}
\hline Algorithm & $\begin{array}{c}\text { Original Size } \\
\text { in } \mathrm{Kb} \\
\end{array}$ & $\begin{array}{c}\text { Compressed } \\
\text { Size in } \mathrm{Kb} \\
\end{array}$ \\
\hline EZW & 150 & 52 \\
\hline ASWDR & 150 & 46 \\
\hline $\begin{array}{l}\text { SPIHIT (True Color } \\
\text { image) }\end{array}$ & 150 & 38 \\
\hline SPIHIT (Gray Scale) & 150 & 26 \\
\hline STW & 150 & 23.4 \\
\hline
\end{tabular}

\section{ADVANTAGES \& DISADVANTAGES OF IMAGE COMPRESSION}

\subsection{Advantages}

1. Format of picture has been being used following long time and is to a great degree compact.

2. Format of image is compatible with verging on every image processing application.

3. Format of image is compatible with most of the hardware component e.g printers etc; therefore it is very easy to print the images in JPEG format.

4. JPEG format can be used to store high resolution fast moving images which would be blur in other image formats because owing to their small size, JPEG images can be stored quickly from a camera to storage device.

5. Size of JPEG images can be decreased and compressed which makes this document design reasonable for exchanging images over the web since it devours less data transfer capacity. A JPEG picture can be compressed down to $5 \%$ of its unique size.

\subsection{Disadvantages}

1. Compression procedure is a lossy compression. Lossy compression implies that after picture is compacted in JPEG design, it loses certain real substance of the images.

2. Quality of Image is decreased after compression attributable to the loss of genuine substance of the images.

3. Image compression is not appropriate for pictures with sharp edges and lines. JPEG images configuration is not equipped for taking care of energized realistic pictures.

4. JPEG images don't bolster layered pictures. Visual planner need to chip away at layered pictures keeping in mind the end goal to control and alter realistic pictures which is impractical with JPEG Images. 
VII. CONCLUSION

The objective of this work was to compress an image. As in many of the devices where the full size images cannot be viewed or are not supported so the compressed images are used. The image compression also helps to save memory, as the size of the compressed image is less than the actual size of the image. In this project we have taken several images, in which original images were converted into compressed images using the various compressing methods. Comparison of various algorithms has been done and it is found that the original image 'wpeppers.jpg' of size $(150 \mathrm{~kb})$ is compressed into a compressed image of size $(23.4 \mathrm{~kb})$ using the STW compression method.

\section{REFERENCES}

1. Christophe, E., C. Mailhes, P. Duhamel (2006), Adaptation of zero trees using signed binary digit representations for 3 dimensional image coding.

2. Misiti, M., Y. Misiti, G. Oppenheim, Wavelets and their applications, ISTE DSP Series.

3. Two Dimensional True Compression /Wavelet Toolbox / http : // in.mathworks.com / help

4. Digital Image Processing using r matlab (Gonzalez)/Chapter 6/Color Image Representation in MATLAB

5. IEEE TRANSACTIONS ON IMAGE PROCESSING, VOL. 17, NO. 12, DECEMBER 2008 Hyper spectral

6. Emmanuel Christophe, Pierre Duhamel, and Corinne Mailhes, Adaptation of Zerotrees Using Signed Binary Digit Representations for 3 Dimensional Image Coding, INTERNATIONAL JOURNAL OF IMAGE AND VIDEO PROCESSING 1

7. Q. Du and J. E. Fowler, image compression using JPEG2000 and principal component analysis, IEEE Geosci. Remote Sens. Lett., vol. 4, no. 4, pp. 201-205, Apr. 2007.

8. Q. Du and J. E. Fowler, Low-complexity principal component analysis for image compression, Int. J. High Performance Comput. Appl.,to be published.

9. D. Van Buren, A high-rate JPEG2000 compression system for space, in Proc. IEEE Aerospace Conf., Mar. 2005, pp. 1-7.

10. P.-S. Yeh, P. Armbruster, A. Kiely, B. Masschelein, G. Moury, C. Schaefer, and C. Thiebaut, The new CCSDS image compression recommendation, presented at the IEEE Aerospace Conf., Mar. 2005.

11. X. Tang, W. A. Pearlman, and J. W. Modestino, image compression using three-dimensional wavelet coding, in Proc. SPIE Image and Video Communications and Processing, 2003, vol. 5022, pp. 1037- 1047.

12. H. Kim, C. Choe, and J. Lee, Fast implementation of 3-D SPIHT using tree information matrix, in Proc. IEEE Int. Geoscience and Remote Sensing Symp., Jul. 2003, vol. 6, pp. 35 